\title{
Protein dynamics: hydration and cavities
}

K. Heremans

\author{
Correspondence \\ K. Heremans \\ Departement of Chemistry \\ Katholieke Universiteit Leuven \\ B-3001 Leuven \\ Belgium \\ E-mail: \\ Karel.Heremans@fys.kuleuven.ac.be \\ Presented at the 3rd International \\ Conference on High Pressure \\ Bioscience and Biotechnology, \\ Rio de Janeiro, RJ, Brazil, \\ September 27-30, 2004.
}

Received January 31, 2005

Accepted May 12, 2005
Departement of Chemistry, Katholieke Universiteit Leuven, Leuven, Belgium

\begin{abstract}
The temperature-pressure behavior of proteins seems to be unique among the biological macromolecules. Thermodynamic as well as kinetic data show the typical elliptical stability diagram. This may be extended by assuming that the unfolded state gives rise to volume and enthalpy-driven liquid-liquid transitions. A molecular interpretation follows from the temperature and the pressure dependence of the hydration and cavities. We suggest that positron annihilation spectroscopy can provide additional quantitative evidence for the contributions of cavities to the dynamics of proteins. Only mature amyloid fibrils that form from unfolded proteins are very resistant to pressure treatment.
\end{abstract}

Key words

- Stability diagram

- Thermodynamics

- High pressure

- Unfolding

- Amyloid fibrils

- Protein dynamics

.....................

\section{Introduction: Life close to the extremes}

Temperature and pressure extremes require different strategies compared to those involved in chemical extremes such as low water activity. In general, organisms cope with the latter by maintaining non-extreme internal conditions or by evolving very effective repair mechanisms (1). Cellular lipids, proteins and nucleic acids are sensitive to high temperatures. Hyperthermophile bacteria have ether lipids instead of the more hydrolysis-sensitive ester lipids in mesophiles. Enzymes from hyperthermophiles show an unusual thermostability in the laboratory and an important aspect of protein chemistry research is to identify the stabilizing principles. Crude cell extracts of hyperthermophiles show the presence of heat-inducible proteins, called chaperones, that assist in the folding of proteins during cellular synthesis. Of special interest is the observa- tion that the inactivation kinetics of microorganisms shows diagrams similar to those of proteins (2). This suggests that proteins are the primary target in the pressure and temperature inactivation of organisms.

Kashefi and Lovley (3) isolated a new archeal species, dubbed strain 121, which grows at $121^{\circ} \mathrm{C}$. This is $8^{\circ} \mathrm{C}$ higher than the previously isolated Pyrolobus fumarii which has a maximum growth rate at $113^{\circ} \mathrm{C}$. Both organisms have survival temperatures of $130^{\circ} \mathrm{C}$ (for $2 \mathrm{~h}$ ) and $121^{\circ} \mathrm{C}$ (for $1 \mathrm{~h}$ ).

The maximum pressure that microbial cells can cope with is of the order of 100 MPa (4,5). But pressure-sensitive processes such as motility, transport, cell division, cell growth, DNA replication, translation, and transcription are affected at much lower pressures. Obviously, a report of the observation of microbial activity at GPa pressure came as a surprise (6). One possible explanation for most of the described effects that was not used in the discussion up to now may come 
from the limited availability of water in the highly concentrated suspension of bacterial cells that were observed in the small volume of the diamond anvil cell.

Another example of the crucial role of water activity in the behavior of organisms under extreme conditions is given by small organisms called Tardigrades. These small animals shrink into a special state when the humidity of the surroundings decreases. In that state they can survive temperature from $-253^{\circ} \mathrm{C}$ up to $151^{\circ} \mathrm{C}$ and pressures up to 600 MPa (7). Under normal conditions they are killed at $200 \mathrm{MPa}$. This behavior is quite similar to that of bacterial spores and dry proteins in which pressures of more than 1 $\mathrm{GPa}$ are not able to provoke any changes $(8,9)$. The fact that the Tardigrades can undergo a transformation to an extreme dry state may be much more exceptional than the fact that they are resistant to extremes of temperature and pressure in the dry state.

Finally, it should be pointed out also that there is no agreement on whether the survival of organisms is a problem of protein stability rather than of chemical stability of small organic molecules.

\section{The protein stability diagram}

The pioneering work in high pressure protein research is that of Bridgman (10) who observed that a pressure of several 100 MPa will give the egg white an outlook similar, but not identical, to that of a cooked egg. Since Bridgman worked on various

Figure 1. The elliptical temperature-pressure stability phase diagram characteristic of proteins. Note the thermodynamic similarities between the cold and pressure unfolding and the contrast with heat unfolding. The possible volume-driven (L1-L2) and enthalpy-driven (L2-L3) transitions are discussed in the text. Modified from Ref. 14. pure substances, he may not have realized that egg white is basically an aqueous solution of a protein in which water plays a vital role in the observed pressure effect. He also made the unexpected observation that the ease of the pressure-induced coagulation increases at low temperatures. In other words, he observed a negative activation energy for a chemical process.

The modern area starts with the seminal paper by Suzuki (11) on the stability of ovalbumin and hemoglobin. Hayashi et al. (12) analyzed the effect of pressure on egg yolk. It is well known from the preparation of a hard boiled egg that the yolk becomes solid at a slightly higher temperature than the white. The reverse is true for the effect of pressure. It is now clear that these observations are the consequence of the unique behavior of proteins.

\section{The stability diagram: thermodynamic aspects}

The first systematic observations of the behavior of proteins with respect to temperature and pressure came from the kinetic studies of Suzuki (11) and the thermodynamic work of Hawley (13). Of particular interest is the fact that the effects of heat, cold and pressure on proteins are interconnected.

The elliptic phase diagram is shown in Figure 1. The shape originates from a Taylor expansion of the free energy difference between the unfolded and the native state of the protein as a function of temperature and pressure, and the second order terms make a significant contribution. Thus, the volume change is not only pressure but also temperature dependent:

$\Delta \mathrm{V}(\mathrm{p}, \mathrm{T})=\Delta \mathrm{V}^{\mathrm{o}}+\Delta \alpha\left(\mathrm{T}-\mathrm{T}_{0}\right)-\Delta \beta\left(\mathrm{p}-\mathrm{p}_{0}\right)$

$\Delta \mathrm{V}^{\mathrm{o}}$ refers to reference conditions. In this equation $\Delta \beta$ is the compressibility factor difference and $\Delta \alpha$ the difference of the ther- 
mal expansion factor between the denatured and native state. The mathematical implications and assumptions that are usually made in the analysis of the data have been recently discussed (14). The physics behind the elliptic phase diagram can be related to the degree of correlation between the enthalpy and volume change of the unfolding transition (15).

The diagram shown in Figure 1 makes it clear that pressure stabilizes the protein against thermal unfolding. Considering the correlation between the enthalpy and volume changes between the cold and the pressure unfolding and the difference with the heat unfolding, one may expect conformational similarities between the cold- and pressure-denatured state and a quite different conformation for the heat-denatured state. This has indeed been observed with infrared spectroscopy for myoglobin (16). On the other hand, in several proteins a lowering of the transition temperature has been observed at higher pressure (13). In this case one does expect no difference between the pressureand temperature-induced transitions since the same thermodynamic conditions prevail, i.e., the enthalpy change is positive and the volume change is negative. Such a situation has been observed for ribonuclease for which the infrared spectra of the heat and pressure unfolded states are similar (17).

Dubins et al. (18) have recently shown for cytochrome $\mathrm{c}$ that for the native-to-unfolded transition the native conformation is stabilized by pressure, whereas for the molten globule-to-unfolded transition the molten globule conformation, at $\mathrm{pH} 2$, is destabilized. Whether this applies to other proteins remains to be determined but the observation suggests that the assumption of the two state unfolding, which is at the basis of the phase diagram, might not be generally valid. Various spectroscopic studies also suggest that the conformation of the temperature- and pressure-unfolded protein are not similar. Fluorescence spectroscopy suggests that the temperature-induced unfolding can be presented as an exposure of the protein core to water, whereas the pressure-induced unfolding results from the pressure-induced penetration of water into the protein matrix followed by a disruption of the chain-chain interactions (19).

\section{Water-soluble synthetic polymers}

The re-entrant phase diagram of proteins has also been observed for a number of water-soluble polymers (20). In most cases the ability to form hydrogen bonds with the solvent is located in the side chains whereas the main chain is expected to be responsible for the hydrophobic interactions. At low temperature and pressure the polymers are in the random coil state (homogeneous one-phase region) and phases separate at high temperature and/or pressure (heterogeneous twophase region). It is of interest to note that some polymers show considerable aggregation in the two-phase region. This can be reduced by the addition of detergents without noticeable effect on the temperature or the pressure at which the transition takes place.

The introduction of polymer-water as well as water-water interactions is important in the modelling of the temperature-concentration behavior of poly(ethylene oxide) (21). This suggests that the strong and highly directional hydrogen bond formation in these systems seems to be as important as the hydrophobic interactions. One would expect high pressure simulations to support this hypothesis.

The shape of the gelatinization diagram of starch as obtained by infrared spectroscopy is also very similar to that of proteins (22). This is quite remarkable given the major differences in composition between these polymers. We note also the important role of water in the gelatinization process. In ethanol no pressure-induced swelling of starch is observed. 
Liquid-liquid transitions in protein solutions?

The re-entrant or Tammann loop-shape phase diagram as observed in proteins and water-soluble polymers is also found in solvent-free systems and has been connected to exothermic disordering (23). In two cases, the nematic-smectic A transitions in liquid crystals and the phase behavior of a crystalline polymer, poly(4-methyl-pentene-1), the phase behavior can be understood by considering the vibrational and configurational contributions to the volume and the entropy of the material in the various phases. Inverse melting, another expression for the same phenomena, has now been computer modeled with a statistical mechanical model (24). However, the challenge remains to simulate the crucial role of water in the phase diagram of water-based systems.

The theoretical and experimental interest in polymorphic phase transitions in liquids and glasses in solvent-free systems such as phosphorous and polymers (25) raises the interesting question of whether similar so-called liquid-liquid transitions might occur in solutions of proteins and water-soluble polymers. Of course, one would expect experimental difficulties in detecting these liquid-liquid transitions because of the small changes in thermodynamic parameters compared to liquidgas and liquid-solid transitions. But the suggestion that the most promising candidates are systems with locally tetrahedral molecular structure such as $\mathrm{C}, \mathrm{SiO}_{2}$ and $\mathrm{H}_{2} \mathrm{O}$ (26) makes aqueous solutions of biopolymers with the predominant role of highly directional hydrogen bonding, good candidates.

Figure 1 includes two possible loci where the so-called liquid-liquid transitions might be expected in the case of unfolded proteins and phase-separated water-soluble polymers. A volume-driven or pressure-induced transition could be observed between the points L1 and L2. Kunugi and co-workers (20) of the Kyoto Institute of Technology reported on such a transition in the case of the hydrogel of poly $(\mathrm{N}$ - vinylisobutyramide). A possible source for such a transition is the effect of pressure on the aggregated state of unfolded proteins $(14,27)$. An enthalpy-driven or a temperature-induced transition at high pressure might also be expected in these systems but has not been reported so far. Note that such transitions are not expected to take place in proteins where only part of the upper part of the diagram is visible as is the case for ribonuclease $(13,17)$.

In nonaqueous systems such as in liquid phosphorous, the low pressure phase consists of a molecular fluid whereas at high pressure a polymeric liquid exists (28). In the case of poly(4-methyl-1-pentene), a nonlinearity of the specific volume of the polymer in the liquid phase gives rise to a reentrant phase diagram with a high temperature and a high pressure maximum (29).

\section{The stability diagram: molecular aspects}

A molecular interpretation of the thermodynamic aspects of the stability diagram may be based on a model for the partial molar volume of a protein. Kauzmann (30) assumed the additivity of the following contributions:

$\mathrm{V}_{\text {protein }}=\mathrm{V}_{\text {atom }}+\mathrm{V}_{\text {cavities }}+\Delta \mathrm{V}_{\text {hydration }}$

Assuming that the volume of the atoms is temperature and pressure independent, it follows that the thermal expansion and the compressibility contain terms for the temperature and pressure dependence of the cavities and the hydration. Unless one has a method that only probes the hydration or the cavities, it will be difficult to make a quantitative estimate of their contributions. Note also the assumption of the additivity of the solvent and the contribution of the hydrated protein to the determination of the thermal expansivity and the compressibility.

\section{Molecular aspects: hydration}

The role of water in the folding and un- 
folding of proteins is very clear from the experimental fact that dry proteins cannot unfold either by temperature or pressure (8). This also explains the pressure and temperature sensitivity of vegetative cells when compared to bacterial spores. It may also explain the extreme resistance to pressure inactivation of prions $(31,32)$.

On the basis of the negative activation energies obtained for the denaturation of proteins under high pressure conditions, Suzuki (11) proposed the insertion of water as the first step in the pressure-induced unfolding of proteins

$$
\text { Protein }+\mathrm{nH}_{2} \mathrm{O} \leftrightarrow \mathrm{P}\left(\mathrm{H}_{2} \mathrm{O}\right)_{\mathrm{n}} \rightarrow \mathrm{P}_{\mathrm{D}}
$$

Computer simulations have also shown that water penetration is the underlying mechanism for pressure-induced unfolding (33, 34). A more detailed picture of protein hydration is obtained from X-ray and neutron scattering experiments and molecular dynamics simulations (35). For lysozyme it was found that the first hydration shell (ca. $3-\AA$ thick) is $15 \%$ denser than bulk water. Smolin and Winter (36) concentrated their efforts on the properties of water at the surface of native staphylococcal nuclease. Taking the excluded volume effect into account, they found that the density is about $0.3-0.6 \%$ higher than the bulk water. Their analysis of the water structure in terms of rings of hydrogen-bonded water molecules points to the strong influence of the chemical and topographical properties of the protein surface.

The most frequently used method to obtain compressibilities of proteins in dilute solution is ultrasound (37). The interpretation of the data is based on the additivity of the compressibilities of the solvent and the solute. If, however, one assumes that the sound propagation of the components is additive, then this gives rise to the conclusion that the contribution of hydration is usually overestimated (38). Computer simulations indicate that the experimental compressibilities obtained from ultrasound can account for the intrinsic compressibility (39). Other computer simulations show, however, that the contribution of hydration is predominant (40). It is clear that we need additional input from experimental data to resolve the discrepancy between these results. Similar considerations apply to a new approach that measures the thermal expansion of solutions with pressure perturbation calorimetry (41). Unfortunately no computer simulations are available which would allow an estimate of the contributions of hydration and cavities from temperature changes. The additivity of volumes, compressibility and thermal expansion may also be viewed from a statistical mechanical treatment of the volume fluctuations (42). When the coupling between the fluctuations of the solvent and the protein is strong, as may be expected in hydrogen-bonded systems, then the additivity rule does not apply.

\section{Molecular aspects: cavities}

The functional role of cavities has been advocated on several occasions in the literature. Richards (43) has emphasized their role in the dynamic behavior of proteins. Gekko and Hasegawa (44) have attributed the lower compressibility of lysozyme at lower temperature in water to increased hydration. Silva and Weber (45) have pointed out the analogy between the volume changes of protein-protein interactions and protein unfolding and have attributed a role to the imperfect packing at intersubunit surfaces. Fusi et al. (46) created a cavity in the interior of a small DNA-binding protein and observed a drastic decrease in temperature as well pressure stability. Silva et al. (47) have recently reviewed the role of cavities in a number of biochemical systems, with the conclusion that their role has been underestimated so far.

An estimate of the effects of temperature 
and pressure on the size of the cavities may be obtained from the structural changes of proteins via NMR spectroscopy. This has been done for hen egg white lysozyme as a function of pressure (48) and for human lysozyme as a function of temperature (49). Some data are presented in Table 1. Despite the considerable progress that has been made in the quantitative characterization of cavities, there is a need for an independent experimental approach to quantify the temperature and pressure dependence of the size of cavities in proteins.

\section{Positron annihilation lifetime spectroscopy}

A possible source of new experimental information on the role of cavities in the compressibility and thermal expansion of proteins is positron annihilation lifetime spectroscopy, which is being used extensively as a direct probe for free-volume in polymers and other materials. The size of the positronium is similar to that of the hydrogen atom and the lifetime of the free ortho-positronium is $140 \mathrm{~ns}$. The lifetime depends on the size of molecular cavities but also on the availability of electrons for pick-off annihilation (50).

For the analysis of the protein in water we need data on the temperature and pressure dependence of the annihilation rates of water. These data show that the activation energy and volume are compatible with the dependence of the surface tension on these variables. The analysis of the protein data is further complicated by the assumptions that have to be made about the role of the water

Table 1. Effects of pressure and temperature on the total volume of the cavities in proteins as obtained from NMR and molecular dynamics (MD) simulations.

\begin{tabular}{llcl} 
Protein & Total cavity volume (conditions) & Method & Ref. \\
\hline Hen lysozyme & $1187 \mathrm{~A}^{3}(30 \mathrm{bar})-1129 \mathrm{~A}^{3}(2 \mathrm{kbar})$ & NMR & 48 \\
Human lysozyme & $855 \mathrm{~A}^{3}\left(4^{\circ} \mathrm{C}\right)-1302 \mathrm{~A}^{3}\left(35^{\circ} \mathrm{C}\right)$ & NMR & 49 \\
Hen lysozyme & $5266 \mathrm{~A}^{3}(1 \mathrm{bar})-4036 \mathrm{~A}^{3}(5 \mathrm{kbar})$ & $\mathrm{MD}$ & 40 \\
\hline
\end{tabular}

that is closely connected with the protein surface. The analysis is in progress in our laboratory.

\section{Intermolecular interactions of unfolded proteins}

The connection between protein folding and the misfolding that occurs under certain conditions and that gives rise to specific fibril formation is now one of the most fascinating topics of protein research (51). The aggregation of misfolded proteins seems to be a common feature of many proteins, and some of them may lead to what is now commonly called conformational diseases. Highly soluble proteins are converted to insoluble filamentous fibrils that are rich in Bpleated structures. Pressure is a very valuable tool for the study of the mechanism of aggregation $(52,53)$.

Most biophysical investigations of fibril formation have attempted to elucidate the structural events that occur during amyloid fibril assembly. A complementary approach is to investigate the stability of amyloid fibrils, a strategy analogous to the study of protein unfolding that has provided many details regarding the forces that drive protein folding and stabilize the native fold.

Recently, we studied the effects of hydrostatic pressure on the stability of amyloid fibrils formed from the transthyretin 105115 peptide $\left(\right.$ TTR $\left._{105-115}\right)$ using Fourier transform infrared spectroscopy. The peptide corresponds to $\beta$-strand $\mathrm{G}$ of the protein and has been shown to readily form amyloid fibrils in vitro. Its small size makes it a useful model system. Information on the morphology of the species was obtained by atomic force microscopy. More experimental details can be found elsewhere (32). The species formed early in the aggregation process were dissociated by relatively low hydrostatic pressure (220 MPa), whereas mature fibrils were pressure insensitive up to 1.3 $\mathrm{GPa}$, as shown in Figure 2. This stability of 
the mature fibrils is consistent with a fibril structure in which there is an extensive hydrogen bond network in a tightly packed environment from which water is excluded. The fact that early aggregates can be dissociated by low pressure suggests, however, that hydrophobic and electrostatic interactions are the dominant factors stabilizing the species formed in the early stages of fibril formation.

Previous study by our group has shown that insulin fibrils are also stable to pressures of at least 1.2 GPa (54). Foguel and coworkers (55), on the other hand, found that amyloid fibrils of full-length TTR and $\alpha$ synuclein can be dissociated in the pressure range of 200-300 MPa. It appears, therefore, that there are significant differences in the stability to pressure of amyloid fibrils formed by different proteins as well as by the same protein at various stages of the aggregation process. High pressure will shift a system towards the state that occupies the smallest volume. Cavities, packing defects and changes in hydration are expected to be major contributors to volume changes and their presence will make the system more susceptible to pressure unfolding/dissociation. The observed pressure insensitivity of the mature fibrils of the TTR peptide, therefore, suggests that these effects are largely absent, a result consistent with the conclusions from X-ray data for a microcrystal of an amyloidogenic peptide that the $\beta$-sheets are densely packed and exclude water (56). Further evidence for the absence of water in the fibrils comes from unpublished hydrogen-deuterium exchange experiments on insulin fibrils that show that the exchange is slow even at high pressures. Interestingly, a similar difference in the pressure stability of early and mature aggregates has been observed for the amorphous aggregates of myoglobin (57) and an amyloid-like structure of the prion protein obtained by thermal treatment (53).

The high stability of the mature fibrils is reminiscent of the stability of bacterial spores and dry proteins (8). In all of these cases, the absence of water is the crucial factor. One would therefore also expect a high temperature stability. This has indeed been observed for the amorphous aggregates of myoglobin (58). Another possible source of the high pressure stability may be the presence of $\beta$ helices in amyloid fibrils. It is well known that enzymes that contain $\beta$-helices are very pressure stable (59).

\section{Conclusions}

From the various aspects of high pressure and high temperature studies on proteins that we have considered in this review, it seems that the most crucial one is the highly directional interaction capacity of hydrogen bonds. Extending the phase diagram along the lines that are being suggested for other systems with directional interactions, we predict the possibility of two additional transitions, one driven by volume at high temperature and the other driven by enthalpy at high pressure.

As to the contributions of the hydration and the cavities, most models assume the additivity of thermodynamic quantities such as volume, compressibility and thermal expansion. A quite different picture emerges if

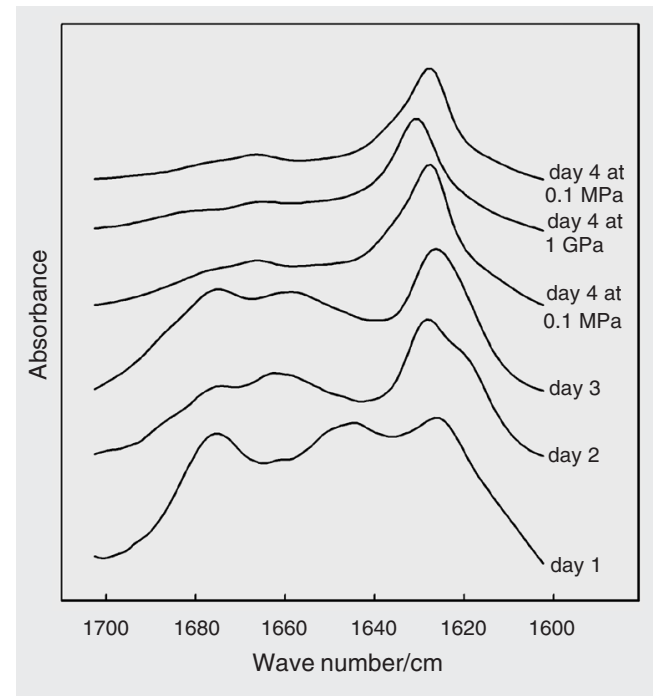

Figure 2. The amide l' region of the FTIR spectrum of transthyretin 105-115 peptide as a function of time and pressure. At day 1 , the pressure strongly affects the spectrum (data not shown). At day 4 , pressure effects are only affecting the vibrations of the hydrogen-bonded $\mathrm{C}=\mathrm{O}$ groups of the peptide bond, indicating no substantial changes in conformation of the protein. Effects are reversible as indicated. High hydrostatic pressure was generated in a diamond anvil cell. 
one considers the coupling between the statistical mechanical fluctuations that are related to these quantities. In view of the predominant role of hydrogen bonding, cavities and hydration may be also considered, up to a certain extent, as two sides of the same phenomenon. A new experimental approach, positron annihilation spectroscopy, might help obtain more quantitative information on the role of the cavities.

Pressure studies have been of special interest in relation to protein unfolding and aggregation. The central question remains to what extent these data apply to the highly crowded medium of the cell. Pressure studies on cellular systems should be of great help in this respect (60).

\section{Acknowledgments}

The discussions with numerous colleagues and friends on various aspects of high pressure and high temperature behavior of biomolecules are highly appreciated.

\section{References}

1. Rothschild LJ \& Mancinelli RL (2001). Life in extreme environments. Nature, 409: 1092-1101.

2. Hashizume C, Kimura K \& Hayashi R (1995). Kinetic-analysis of yeast inactivation by high-pressure treatment at low temperatures. Bioscience, Biotechnology, and Biochemistry, 59: 1455-1458.

3. Kashefi K \& Lovley DR (2003). Extending the upper temperature limit for life. Science, 301: 934.

4. Yayanos AA (1986). Evolutional and ecological implications of the properties of deep-sea barophilic bacteria. Proceedings of the $\mathrm{Na}$ tional Academy of Sciences, USA, 83: 9542-9546.

5. Bartlett $\mathrm{DH}$ (2002). Pressure effects on in vivo microbial processes. Biochimica et Biophysica Acta, 1595: 367-381.

6. Sharma A, Scott JH, Cody GD et al. (2002). Microbial activity at gigapascal pressures. Science, 295: 1514-1516.

7. Seki K \& Toyoshima M (1998). Preserving tardigrades under pressure. Nature, 395: 853-854.

8. Goossens K, Smeller L, Frank J et al. (1996). Pressure-tuning spectroscopy of bovine pancreatic trypsin inhibitor: A high pressure FT-IR study. European Journal of Biochemistry, 236: 254-262.

9. Sojka B \& Ludwig H (1997). Effects of rapid pressure changes on the inactivation of Bacillus subtilis spores. Pharmazeutische Industrie, 59: 436-438.

10. Bridgman PW (1914). The coagulation of albumen by pressure. Journal of Biological Chemistry, 19: 511-512.

11. Suzuki K (1960). Studies on the kinetics of protein denaturation under high pressure. Review of Physical Chemistry of Japan, 29: 91-98.

12. Hayashi R, Kawamura Y, Nakasa T et al. (1989). Application of highpressure to food-processing - pressurization of egg-white and yolk, and properties of gels formed. Agricultural and Biological Chemistry, 53: 2935-2939.

13. Hawley SA (1971). Reversible pressure-temperature denaturation of chymotrypsinogen. Biochemistry, 10: 2436-2442.

14. Smeller $L$ (2002). Pressure-temperature phase diagrams of biomolecules. Biochimica et Biophysica Acta, 1595: 11-29.

15. Lesch $\mathrm{H}$, Hecht $\mathrm{C}$ \& Friedrich J (2004). Protein phase diagrams: the physics behind their elliptic shape. Journal of Chemical Physics, 121: 12671-12675.

16. Meersman F, Smeller L \& Heremans K (2002). Comparative Fourier transform infrared spectroscopy of cold-, pressure- and heat-induced unfolding and aggregation of myoglobin. Biophysical Journal,
82: $2635-2644$.

17. Torrent J, Rubens $\mathrm{P}$, Ribo $\mathrm{M}$ et al. (2001). Pressure versus temperature unfolding of ribonuclease A: An FTIR spectroscopic characterization of 10 variants at the carboxy-terminal site. Protein Science, 10: 725-734.

18. Dubins DN, Filifil R, MacGregor Jr RB et al. (2003). Volume and compressibility changes accompanying thermally-induced nativeto-unfolded and molten globule-to-unfolded transitions of cytochrome c: A high pressure study. Biochemistry, 42: 8671-8678.

19. Lesch H, Stadlbauer H, Friedrich J et al. (2002). Stability diagram and unfolding of a modified cytochrome c: What happens in the transformation regime? Biophysical Journal, 82: 1644-1653.

20. Kunugi S, Takano K, Tanaka $\mathrm{N}$ et al. (1997). Effects of pressure on the behavior of the thermoresponsive polymer poly( $\mathrm{N}$-vinylisobutyramide) (PNVIBA). Macromolecules, 30: 4499-4501.

21. Dormidontova EE (2002). Role of competitive PEO-water and water-water hydrogen bonding in aqueous solution PEO behavior. Macromolecules, 35: 987-1001.

22. Rubens P \& Heremans K (2000). Pressure-temperature gelatinization phase diagram of starch: an in situ FTIR study. Biopolymers, 54 524-530.

23. Johari GP (2001). The Tammann phase boundary, exothermic disordering and the entropy contribution change on phase transformation. Physical Chemistry Chemical Physics, 3: 2483-2487.

24. Feeney MR, Debenedetti PG \& Stillinger FH (2003). A statistical mechanical model for inverse melting. Journal of Chemical Physics, 119: 4582-4591.

25. Yarger JL \& Wolf GH (2004) Polymorphism in liquids. Science, 306: 820-821.

26. McMillan PF (2004). Polyamorphic transformations in liquids and glasses. Journal of Materials Chemistry, 14: 1506-1512.

27. Meersman F, Smeller L \& Heremans K (2005). Exploring the pressure-temperature state diagram of myoglobin. Helvetica Chimica Acta (in press).

28. Monaco G, Falconi S, Crichton WA et al. (2003). Nature of the firstorder phase transition in fluid phosphorus at high temperature and pressure. Physical Review Letters, 90: 255701.

29. van Ruth NJL \& Rastogi S (2004). Nonlinear changes in specific volume. A route to resolve an entropy crisis. Macromolecules, 37 8191-8194.

30. Kauzmann W (1959). Some factors in the interpretation of protein 
denaturation. Advances in Protein Chemistry, 14: 1-63.

31. Garcia AF, Heindl P, Voigt $\mathrm{H}$ et al. (2004). Reduced proteinase $\mathrm{K}$ resistance and infectivity of prions after pressure treatment at 60 degrees C. Journal of General Virology, 85: 261-264.

32. Dirix C, Meersman F, MacPhee CE et al. (2005). High hydrostatic pressure dissociates early aggregates of TTR(105-115), but not the mature amyloid fibrils. Journal of Molecular Biology (in press).

33. Wroblowski B, Diaz JF, Heremans K et al. (1996). Molecular mechanics of pressure-induced conformational changes in bovine pancreatic trypsin inhibitor. Proteins, 25: 446-455.

34. Hummer G, Garde S, Garcia AE et al. (1998). The pressure dependence of hydrophobic interactions is consistent with the observed pressure denaturation of proteins. Proceedings of the National Academy of Sciences, USA, 95: 1552-1555.

35. Merzel F \& Smith JC (2002). Is the first hydration shell of lysozyme of higher density than bulk water? Proceedings of the National Academy of Sciences, USA, 99: 5378-5383.

36. Smolin N \& Winter R (2004). Molecular dynamics simulations of staphylococcal nuclease: Properties of water at the protein surface. Journal of Physical Chemistry B, 108: 15928-15937.

37. Chalikian TV (2003). Volumetric properties of proteins. Annual Review of Biophysics and Biomolecular Structure, 32: 207-235.

38. Pfeiffer H \& Heremans K (2002). Apparent sound velocity of lysozyme in aqueous solutions. Chemical Physics Letters, 361: 226230.

39. Dadarlat VM \& Post CB (2003). Adhesive-cohesive model for protein compressibility: an alternative perspective on stability. Proceedings of the National Academy of Sciences, USA, 100: 14778-14783.

40. Marchi M (2003). Compressibility of cavities and biological water from Voronoi volumes in hydrated proteins. Journal of Physical Chemistry B, 107: 6598-6602.

41. Ravindra R, Royer C \& Winter R (2004). Pressure perturbation calorimetric studies of the solvation properties and the thermal unfolding of staphylococcal nuclease. Physical Chemistry Chemical Physics, 6: 1952-1961.

42. Pfeiffer $H$ \& Heremans $K$ (2005). The sound velocity in ideal liquid mixtures from thermal volume fluctuations. ChemPhysChem, 6: 697705.

43. Richards FM (1979). Packing defects, cavities, volume fluctuations, and access to the interior of proteins - including some general comments on surface-area and protein-structure. Carlsberg Research Communications, 44: 47-63.

44. Gekko K \& Hasegawa $Y$ (1989). Effect of temperature on the compressibility of native globular-proteins. Journal of Physical Chemistry, 93: 426-429.

45. Silva JL \& Weber G (1993). Pressure stability of proteins. Annual Review of Physical Chemistry, 44: 89-113.

46. Fusi P, Goossens K, Consonni R et al. (1997). The extremely heat- and pressure-resistant $7 \mathrm{kDa}$ protein $\mathrm{P} 2$ from the archaeon Sulfolobus solfataricus is dramatically destabilized by a single amino acid substitution. Proteins, 29: 381-390.

47. Silva JL, Foguel D \& Royer C (2001). Pressure provides new insights into protein folding, dynamics and structure. Trends in Biochemical Sciences, 26: 612-618.

48. Refaee M, Tezuka T, Akasaka K et al. (2003). Pressure-dependent changes in the solution structure of hen egg-white lysozyme. Journal of Molecular Biology, 327: 857-865.

49. Kumeta H, Miura A, Kobashigawa $Y$ et al. (2003). Low-temperatureinduced structural changes in human lysozyme elucidated by threedimensional NMR spectroscopy. Biochemistry, 42: 1209-1216.

50. Süvegh K, Vértes A \& Hyodo T (1999). Positronium as a sensitive detector of changes in molecular structure. Advances in Molecular Structure Research, 5: 313-357.

51. Dobson CM (2003). Protein folding and misfolding. Nature, 426 884-890.

52. Torrent J, Marchali S, Tortora P et al. (2004). High pressure, an alternative approach to understand protein unfolding diseases. Cellular and Molecular Biology, 50: 377-385.

53. Cordeiro $\mathrm{Y}$, Kraineva J, Ravindra $\mathrm{R}$ et al. (2004). Hydration and packing effects on prion folding and beta-sheet conversion: highpressure spectroscopy and pressure perturbation calorimetry studies. Journal of Biological Chemistry, 279: 32354-32359.

54. Dirix C, Meersman F, Smeller L et al. (2002). Unfolding and fibrillogenesis of insulin: temperature, pressure and chemistry. High Pressure Research, 22: 733-736.

55. Foguel D, Suarez MC, Ferrão-Gonzales AD et al. (2003). Dissociation of amyloid fibrils of alpha-synuclein and transthyretin by pressure reveals their reversible nature and the formation of waterexcluded cavities. Proceedings of the National Academy of Sciences, USA, 100: 9831-9836.

56. Balbirnie M, Grothe R \& Eisenberg DS (2001). An amyloid-forming peptide from the yeast prion Sup35 reveals a dehydrated B-sheet structure for amyloid. Proceedings of the National Academy of Sciences, USA, 98: 2375-2380.

57. Smeller L, Rubens P \& Heremans K (1999). Pressure effect on the temperature-induced unfolding and tendency to aggregate of myoglobin. Biochemistry, 38: 3816-3820.

58. Meersman F \& Heremans K (2003). Temperature-induced dissociation of protein aggregates: accessing the denatured state. Biochemistry, 42: 14234-14241.

59. Ly-Nguyen B, Van Loey AM, Smout C et al. (2003). Mild-heat and high-pressure inactivation of carrot pectin methylesterase: A kinetic study. Journal of Food Science, 68: 1377-1383.

60. Fernandes PMB, Domitrovic T, Cao CM et al. (2004). Genomic expression pattern in Saccharomyces cerevisiae cells in response to high hydrostatic pressure. FEBS Letters, 556: 153-160. 